\title{
Summary Abstract: Localization of superlattice electronic states and complex bulk band structures
}

\author{
J. N. Schulman \\ University of Hawaii, Honolulu, Hawaii 96822
}

T. G. McGill

California Institute of Technology, Pasadena, California 91125

(Received 14 May 1980; accepted 20 May 1980)

PACS numbers: $73.40 . \mathrm{Lq}$

The relative lineup of the band structures of the two constituents of a semiconductor superlattice can cause charge carriers to be confined. ${ }^{1,2}$ This occurs when the energy of a superlattice state is located in an allowed energy region of one of the constituents (the "well" semiconductor), but in the band gap of the other (the "barrier" semiconductor). A charge carrier will tend to be confined in the layers made from the semiconductor with the allowed region at that energy. It will have an exponentially decaying amplitude to be found in the semiconductor with a band gap at that energy.

The decay length of the state is a measure of the degree to which the carrier is confined to the semiconductor with the allowed energy region. Short decay lengths in the barrier semiconductor indicate that there is only a small overlap between the well-like wave functions centered in neighboring wells. The effective mass in the direction perpendicular to the superlattice layers will thus be large. Conversely, long decay lengths result in small perpendicular effective masses.

We report the results of a theoretical calculation which determined the decay lengths of superlattice states at the conduction and valence band edges for two superlattice systems: AlAs/GaAs and $\mathrm{CdTe} / \mathrm{HgTe}$. A parameterized tightbinding Hamiltonian was used to calculate bulk band structures for complex values of the wave vector. The complex bulk band structures of the barrier semiconductors in the forbidden energy regions gave dispersion curves of energy vs complex wave vector. The reciprocal of the imaginary part of the wave vector for a state with a specified energy is the decay length of that state in the barrier semiconductor. 3,4

The procedure followed to determine the superlattice state decay lengths was first to find the superlattice valence and conduction band edge energies from a superlattice tightbinding calculation, and then to find the intersection of these energies with the complex bulk band structure of the barrier semiconductor. The decay lengths of the bulk states at these energies are, to a good approximation, the decay lengths of the superlattice states. ${ }^{3,4}$ The influence of the decay lengths on effective masses in the direction perpendicular to the superlattice layers was investigated by comparing calculated effective masses vs layer thickness for the two superlattice systems.

In addition, the InAs/GaSb superlattice was studied. This system is of particular interest because the conduction band edge state is localized in the InAs layers while the valence band edge state is localized in the GaSb layers. ${ }^{2}$ Thus both semiconductors act as barriers. For this system a simple twoband approximation was used to calculate the complex bulk band structures. ${ }^{4}$ Only the band gap and bulk effective masses were needed in this calculation. The energies of the superlattice states, however, cannot be found with this method. Our results for all three superlattice systems follow.

In the case of the AlAs/GaAs superlattice, AlAs is the barrier semiconductor. The conduction and valence band edge energies of the superlattice consisting of 20 atomic layers of AlAs alternating with 10 atomic layers of GaAs were found. These two energies intersect the AlAs complex dispersion curves at two points each. There are thus two possible decay lengths for each superlattice state. Group theory arguments eliminate one of the two possibilities, resulting in a unique decay length for each state. The decay length of the valence band maximum state is $7.2 \AA$ and that of the conduction band minimum state is $4.0 \AA$. These values of the decay lengths are in excellent agreement with those determined from the superiattice wave function directly.

The CdTe/HgTe superlattice is more complicated. CdTe is the barrier semiconductor. ${ }^{5}$ Spin-orbit splitting, which was ignored when calculating the AlAs complex band structure, is large and must be included. The group theory arguments no longer apply with the spin-orbit interaction and therefore there is no unique decay length. The conduction band superlattice state in the CdTe layers is found to consist of bulk states with decay lengths of 9.3 and $18.7 \AA$.

The longer decay lengths of the CdTe/HgTe superlatice states as compared with the AlAs/GaAs superlattice states result in more overlap between the welllike wavefunctions centered in neighboring wells. The effective masses of the $\mathrm{CdTe} / \mathrm{HgTe}$ superlattice in the perpendicular direction would therefore be expected to vary more slowly with $\mathrm{CdTe}$ layer thickness than would the AlAs/GaAs superlattice effective masses with AlAs layer thickness. Detailed calculations of these effective masses for varying layer thicknesses support this conclusion.

The complex bulk band structure of $\mathrm{InAs}$ and $\mathrm{GaSb}$ were obtained in a two-band approximation. This approximation was found to give excellent agreement with the more detailed tight-binding calculation when applied to the AlAs/GaAs and $\mathrm{CdTe} / \mathrm{HgTe}$ superlattices. ${ }^{4}$ Again, the spin-orbit interaction in InAs and $\mathrm{GaSb}$ is large and, therefore, a unique decay length cannot be found. Instead, a superlattice state at a certain energy would consist of bulk states with two decay 
lengths, as with the CdTe/HgTe superlattice. Superlattice state energies have been determined by others, although with some disagreement. ${ }^{6-9}$ We estimated the decay lengths of these states using the InAs and GaSb complex band structures.

In summary, we have developed a theoretical technique that is useful in determining the degree of confinement of charge carriers in semiconductor superlattices. The complex band structures of the barrier semiconductor can be investigated to determine the decay lengths of superlattice states and thus the carrier confinement. This technique should be useful in understanding properties which depend on the carrier confinement, such as optical absorption ${ }^{2}$ and transport properties perpendicular to the superlattice layers.
Acknowledgment: Work supported in part by the ARO under Contract No. DAAG29-77-C-0015.

${ }^{1}$ R. Dingle, H. L. Störmer, A. C. Gossard, and W. Wiegmann, Appl. Phys. Lett. 33, 665 (1978).

${ }^{2}$ G. A. Sai-Halasz. L. I. Chang, J. M. Walter, C. A. Chang, and I. Esaki, Solid-State Commun. 27, 935 (1978).

3]. N. Schulman and T. C. McGill, Solid-State Communun, 34, 29 (1980).

$4 \bar{j}$. N. Sehulman and T. C. MeGill (unpublished).

5 J. N. Schuman and T. C. McGill, Appl. Phys. Lett. 34, 663 (1979).

6y. Thm, Pui K. Lam, and Marvin L. Cohen, Phys. Rev. B 20, 120 (1979).

${ }^{7}$ G. A. Sai-Halasz, R. Tsu, and L. Esaki, Appl. Phys. Lett. 30, 651 (1977).

${ }^{8}$ H. Bluyssen, J. C. Maan, P. Wyder, L. Chang, and L. Esaki, Solid-State Commun. 31, 35 (1979).

9R. N. Nucho and A. Madhukar, J. Vac. Sci. Technol. 15, 1530 (1978). 